\title{
Endocrine effects on heart function
}

\author{
M.R. Gamberini, ${ }^{1}$ A. Meloni, ${ }^{2}$ V. Caruso, ${ }^{3}$ M. Capra, ${ }^{4}$ P. Cianciulli, ${ }^{5}$ E. Chiodi, ${ }^{6}$ M. Lombardi, ${ }^{2}$ \\ A. Pepe ${ }^{2}$ \\ ${ }^{1}$ Pediatria, Adolescentologia e Talassemia, Arcispedale "S. Anna", Ferrara, Italy; ${ }^{2}$ Fondazione \\ G.Monasterio CNR-Regione Toscana and Institute of Clinical Physiology, Pisa, Italy; ${ }^{3}$ Unità \\ Operativa Dipartimentale Talassemia, P.O. "S. Luigi-Currò" - ARNAS Garibaldi, Catania, Italy; \\ ${ }^{4}$ Servizio Prevenzione, Diagnosi e Cura Talassemia, Ospedale "G. di Cristina", Palermo, Italy; \\ ${ }^{5}$ Centro Talassemie, Ospedale "Sant'Eugenio Papa", Roma, Italy; 'Servizio Radiologia \\ Ospedaliera-Universitaria, Arcispedale "S. Anna" di Ferrara, Ferrara, Italy
}

\begin{abstract}
Among the factors associated with thalassemic heart disease, endocrine disturbance is also a contributing factor. We present a retrospective, cross sectional study, which aims to establish the prevalence of cardiac complications in thalassaemia major (TM) patients with endocrine complications and to evaluate the influence of endocrine disease on cardiac complications. Endocrinological and cardiological parameters were considered on 957 TM patients who are enrolled in the Myocardial Iron Overload in Thalassemia (MIOT) network in 68 sites in Italy. Patients with pubertal hypogonadism (163 males and 175 females), hypothyroidism (192), diabetes mellitus (87) and hypoparathyroidism (61), were compared according to cardiac complications: global heart $\mathrm{T}^{*}$, cardiac dysfunction, heart failure, arrythmias, pulmonary hypertension and myocardial fibrosis. Control groups were made up according to the age range of patients with the corresponding endocrinopathy. The prevalence of cardiac dysfunction, arrhythmias and heart failure was significantly increased in patients with endocrinopathies. Cardiac complications tended to increase according to the number of endocrinologies affecting the patient.
\end{abstract}

\section{Introduction}

In thalassemia heart diseases are associated with many factors. Iron overload and chronic anemia are the leading causes but a role is also played by other elements such as myocarditis, pulmonary hyper-

Correspondence: M.R. Gamberini, Pediatria, Adolescentologia e Talassemia, Arcispedale "S. Anna", Ferrra, Italy.

Key words: jeart function, endocrine effects.

(C) Copyright M.R. Gamberini et al., 2011

Licensee PAGEPress, Italy

Thalassemia Reports 2011; 1(s2):e19

doi:10.4081/thal.2011.s2.e19

This article is distributed under the terms of the Creative Commons Attribution Noncommercial License (by-nc 3.0) which permits any noncommercial use, distribution, and reproduction in any medium, provided the original author(s) and source are credited.

Parts of this work were presented at the "12th International Conference on Thalassemia and Hemoglobinopathies", Antalya (Turkey), 11-14 May 2011. tension, cirrhosis, defect in antioxidant mechanisms, nutritional deficiencies, genetic factors, premature vascular aging, endocrine diseases. ${ }^{1,2}$ Endocrine diseases and cardiac complications share the same etiologic factors that are chronic anemia and iron overload, 3,4 but the relationship between endocrine diseases and cardiac complications is not well established. In fact, in the literature only case reports regarding cardiac failure secondary to decompensated hypothyroidism and hypoparathyroidism or heart failure associated with growth hormone deficiency are present. ${ }^{5,6}$

Aim of our retrospective and cross sectional study was to establish the prevalence of cardiac complications in thalassemia major (TM) patients with endocrine diseases and to evaluate the influence of endocrine diseases on cardiac complications.

\section{Patients and Methods}

We considered the data of TM patients enrolled in the Myocardial Iron Overload in Thalassemia (MIOT) network, involving 8 cardiac Magnetic Resonance Imaging (MRI) Centres and 68 thalassemia sites in Italy. The MIOT Centres are linked by an online database designed to collect and share patient history, clinical and diagnostic data. The magnetic resonance protocol includes the evaluation of liver and myocardial iron content, heart function and myocardial fibrosis. In all Centres MRI examinations are performed in a standardized fashion by using 1.5T scanners (GE Medical Systems, Milwaukee, WI, USA). ${ }^{7,8}$

A multislice multiecho T2* approach was used to measure myocardial iron overload. ${ }^{9}, 10$ Three short-axis views of the left ventricle (LV) were acquired and the myocardium was divided into 16 segments, according to the American Heart Association (AHA) standardized LV model. ${ }^{11}$ The $\mathrm{T} 2 *$ value on each segment was calculated as well as the global value. Morphological and functional biventricular parameters were quantitatively evaluated in a standard way by SSFP cine images using MASS ${ }^{\circledR}$ software. In order to detect myocardial fibrois, contrast delayed enhanced images were acquired after 10-18 minutes from the intravenous administration of a gadolinium-based contrast medium. Short, vertical, horizontal, and oblique long-axis views were acquired. Delayed enhancement (DE) extent was evaluated visually using a twopoint scale (enhancement absent or present) and considered present when visualized in two different views. ${ }^{12}$

A T2* value of $20 \mathrm{~ms}^{13}$ was used as the normal cut-off for all segments. The normal values of ejection fraction (EF) normalized by sex and age, previously obtained in a cohort of 142 TM patients without cardiac disease and iron overload, ${ }^{14,15}$ were used to define left and right heart dysfunction ( $\mathrm{EF}<$ mean -2 standard deviation). Heart failure was diagnosed by MRI in presence of a LV or right ventricular (RV) EF lower than 4 standard deviations from the normalized mean value and by a positive 
history (clinical symptoms, confirmation by physical examination and treatment). A pulmonary hypertension was diagnosed if the trans-tricuspidal velocity jet was greater than $3.2 \mathrm{~m} / \mathrm{s}$ or the gradient values where greater than $40 \mathrm{mmHg} .{ }^{16}$ The diagnosis of arrhythmias, such as atrial fibrillation, atrial flutter, ventricular or supraventricular tachycardia, and ventricular fibrillation, was demonstrated by the ECG.

All data were analyzed using SPSS v. 13.0 (Chicago, IL) statistical package. All continuous variables were expressed as the mean and standard deviation. The Wilcoxon's signed rank test was applied for the comparison of global heart $\mathrm{T} 2 *$ values. $\chi 2$ testing was performed for non-continuous variables. The univariate logistic regression analysis was used to evaluate the odds ratio (OR) with 95\% CI. Adjustment for age or/and co-morbidity was applied. In all tests, a 2-tailed probability value of 0.05 was considered statistically significant.

\section{Results and Discussion}

Data on 957 TM patients at the first cardiac MRI examination (466 $\mathrm{M}$ and $491 \mathrm{~F}$, mean age $30.8 \pm 8.7$ years) were analyzed.

Pubertal hypogonadism was present in 163 males (age 15-59 years) and in 175 females (age 14-5lyears), hypothyroidism in 192 patients (age 14-50 years; $80 \%$ under l-thyroxine treatment), diabetes mellitus in 87 cases (age 19-51 years; 97\% under insulin treatment), hypoparathyroidism in 61 patients (age 19-51 years; all under Vitamin D treatment).

The prevalence of endocrine complications is shown in Figure 1. No difference between males and females was found.

Control groups were made up according to the range of age found in patients with the corresponding endocrinopathy. For each endocrinopathy, the age and the presence of other associated endocrine diseases were significantly higher in patients having the related endocrinopathy.

The global heart T2* values, the prevalence of cardiac complications and myocardial fibrosis according to endocrine diseases are reported in Table 1. Global heart $\mathrm{T} 2 *$ values were significantly lower in all groups of patients with a specific endocrinopathy, with the exception of hypoparathyroidism. The prevalence of cardiac dysfunction was not significantly different in patients with and without a specific endocrinopthy, but it was near to the statistical significance in males with hypogonadism, in patients with hypothyroidism and diabetes. The overall prevalence of heart failure (by MRI and by patient history) ranged between $14 \%$ in females with hypogonadism and $30 \%$ in diabetic patients, and it was significantly more frequent in patients with than without every considered endocrinopathy. Hyperkinetic arrhythmias,

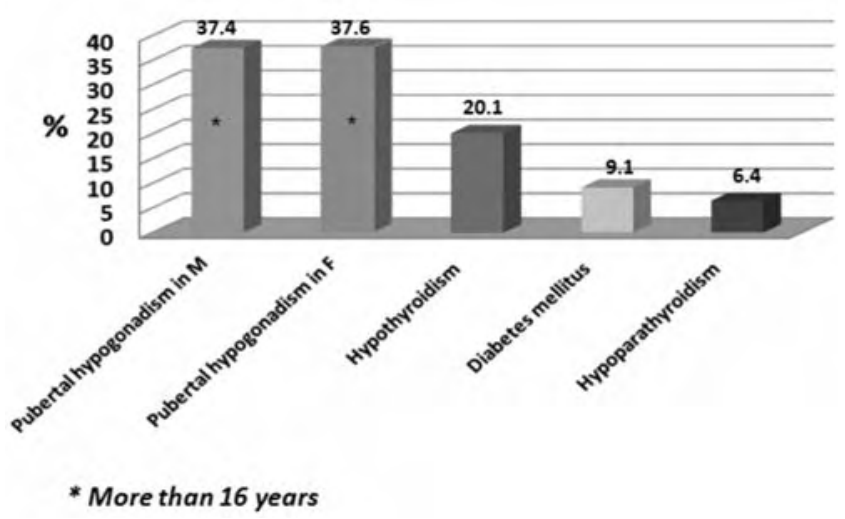

Figure 1. Prevalence of endocrine complications in 957 patients with thalassemia major . mainly supraventricular, were found most frequently than the hypokinetic types. Only hyperkinetic arrhythmias were significantly more prevalent in patients with than without an endocrinopathy. The highest prevalence (18\%) was found in males with hypogonadism and diabetic patients. As for pulmonary hypertension, no significant difference in prevalence between groups was revealed. Cardiac complications, including heart failure, arrhythmias and pulmonary hypertension, were significantly more frequent in patients with than without a specific endocrinopathy (Figure 2). The highest rate of cardiac complications (46\%) was detected in patients with diabetes and the lowest one (21\%) in females with hypogonadism. The prevalence of myocardial fibrosis, ranging from 19 to $30 \%$, was significant higher only in patients with diabetes. Logistic regression analysis relative to the risk of cardiac diseases and co-morbidity, showed that the presence of one, two and more than two versus no endocrine diseases increased the risk for cardiac complications, such as arrhythmias and heart failure. The number of endocrine diseases didn't influence the presence of cardiac dysfunction and myocardial fibrosis. Data are reported in Figure 3. The fact that the patients with a specific endocrinopathy had more cardiac iron content and complications, in particular heart failure and hyperkinetic arrhythmias, than patients without the endocrinopathy confirmed the causative role of iron overload in both endocrine and cardiac complications.

In order to define the impact of the considered endocrinopathies on the development of cardiac diseases, we selected cases without myocardial iron overload (all normal segmental T2* values) (Figure 4). The prevalence of myocardial fibrosis and cardiac complications which were current at the time of the study according to the endocrinopathies is reported in Table 2. Cardiac dysfunction ranged between $12 \%$ in patients with hypoparathyroidism and $43 \%$ in males with hypogonadism. A borderline significance was found when comparing patients with diabetes or hypoparathyroidism versus patients without the specific endocrinopathy. The prevalence of heart failure varied from $7 \%$ in females with hypogonadism to $24 \%$ in patients with diabetes and it was significantly higher only in patients with versus no diabetes. The prevalence of hyperkinetic arrhythmias speckled from the $10 \%$ in females with hypogonadism to the $35 \%$ in patients with diabetes and it was significantly more frequent in all groups having versus not having a specific endocrinopathy. Myocardial fibrosis was more prevalent in patients with than without a specific endocrinopathy, but the difference was significant only considering the diabetes. The probability of arrhythmias increased significantly in presence of two or more associated endocrine diseases (Figure 3). No effect of endocrine co-morbidity was found on heart failure, fibrosis and cardiac dysfunction. Data regarding the $\mathrm{OR}$ corrected for age and co-morbidity are showed in

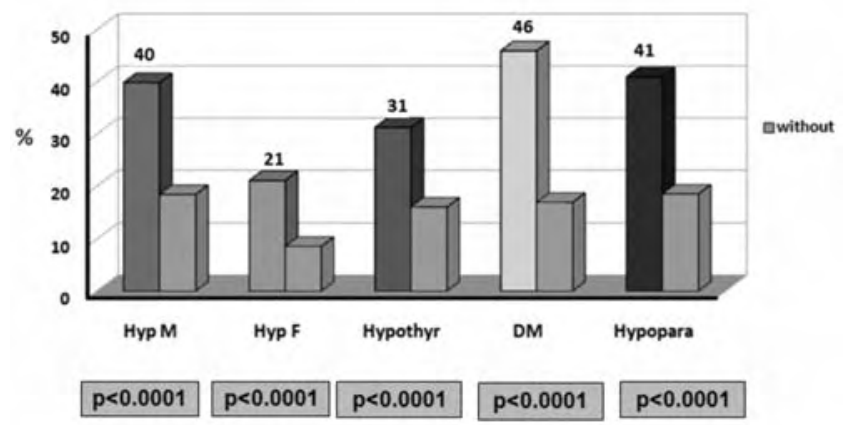

(Hyp $\mathrm{M}=$ pubertal hypogonadism in males; Hyp $\mathrm{F}=$ pubertal hypogonadism in females; Hypthyr=hypothyroidism; DM=diabetes mellitus; Hypopara=hypoparathyroidism).

Figure 2. Prevalence of cardiac complications (heart failure, cardiac dysfunction, arrhythmias) in patients with thalassemia major according to endocrinopathy. 
Table 3. The males with hypogonadism were significantly more likely to have arrhythmias even after adjustment for a single or for both factors (OR 17.21). In females having hypogonadism odds ratio for arrhythmias lose significance after the adjustment for age and co-morbidity. Patients with hypothyroidsm were significantly more likely to have arrhythmias after the correction for age, but not for co-morbidity and for both variables. Comparing patients with and without diabetes, the odds ratio remained significant for arrhythmias after the adjustment for age and co-morbidity, for heart failure only after the adjustment for the presence of another endocrinopathy and for myocardial fibrosis after the correction for both variables. Comparing patients with and without hypoparathyroidism, the odds ratio for arrhythmias lose significance after the correction for both factors. It is important to note that hypoparathyroidism appeared to reduce significantly the risk of cardiac dysfunction. The odds ratio after correction for age and co-morbidity was, in fact, 0.24 . Globally, the analysis performed in patients without cardiac iron overload displayed some impact of the considered endocrinopathy on cardiac complications. Hypogonadism in females

Table 1. Global Heart T2* values, prevalence of cardiac complications and myocardial fibrosis according to endocrine diseases in patients with thalassemia major.

\begin{tabular}{|c|c|c|c|c|c|c|c|c|c|c|}
\hline & \multicolumn{2}{|c|}{$\begin{array}{l}\text { Pubertal } \\
\text { hypogonadism } \\
\text { in malos }\end{array}$} & \multicolumn{2}{|c|}{$\begin{array}{c}\text { Pubertal } \\
\text { hypogonadism } \\
\text { in fermales }\end{array}$} & \multicolumn{2}{|c|}{ Hypothyroidism } & \multicolumn{2}{|c|}{ Diabetes mellitus } & \multicolumn{2}{|c|}{ Hypoparathyroidism } \\
\hline & with & without & with & without & with & without & with & without & with & without \\
\hline $\begin{array}{l}\text { Number of } \\
\text { patients }\end{array}$ & 163 & 272 & 175 & 290 & 192 & 727 & 87 & 708 & 61 & 798 \\
\hline $\begin{array}{l}\text { Global Heart } \\
\text { T2* (ms) }\end{array}$ & $\begin{array}{l}27.0 \pm \\
13.3\end{array}$ & $\begin{array}{c}30.9 \mathrm{t} \\
11.2\end{array}$ & $\begin{array}{c}25.9 \mathrm{z} \\
12.5\end{array}$ & $\begin{array}{l}29.1 . \\
11.7\end{array}$ & $\begin{array}{c}26.4 \pm \\
12.9\end{array}$ & $\begin{array}{c}29.2 \pm \\
11.9\end{array}$ & \begin{tabular}{|c|}
$25.5 \pm$ \\
12.6
\end{tabular} & $\begin{array}{c}29.2 . \\
11.9\end{array}$ & $\begin{array}{c}26.9 \mathrm{z} \\
12.9\end{array}$ & $\begin{array}{c}28.6 \pm \\
12.1\end{array}$ \\
\hline$p$ & \multicolumn{2}{|c|}{0.008} & \multicolumn{2}{|c|}{0.005} & \multicolumn{2}{|c|}{0.001} & \multicolumn{2}{|c|}{$\frac{1}{0.017}$} & \multicolumn{2}{|r|}{$\frac{1}{n s}$} \\
\hline $\begin{array}{l}\text { Cardiac } \\
\text { dysfunction } \\
\text { (\%) }\end{array}$ & 51 & 43 & 25 & 29 & 41 & 34 & 45 & 35 & 33 & 37 \\
\hline$p$ & \multicolumn{2}{|c|}{ ns } & \multicolumn{2}{|c|}{ ns } & \multicolumn{2}{|c|}{ ns } & \multicolumn{2}{|c|}{0.064} & \multicolumn{2}{|c|}{$\mathrm{ns}$} \\
\hline $\begin{array}{l}\text { Heart failure } \\
\text { (by MRI and by } \\
\text { patient history) } \\
\text { (\%) }\end{array}$ & 27 & 13 & 14 & 7 & 21 & 11 & 30 & 12 & 28 & 13 \\
\hline$p$ & \multicolumn{2}{|c|}{80.0001} & \multicolumn{2}{|c|}{0.010} & \multicolumn{2}{|c|}{$<0.0001$} & \multicolumn{2}{|c|}{$<0.0001$} & \multicolumn{2}{|c|}{$\frac{1}{<0001}$} \\
\hline $\begin{array}{l}\text { Hyperkinetic } \\
\text { arrhythmias } \\
\text { (\%) }\end{array}$ & 18 & 4 & 8 & 1.4 & 14 & 4 & 18 & 6 & 13 & 6 \\
\hline $\mathrm{p}$ & \multicolumn{2}{|c|}{80.0001} & \multicolumn{2}{|c|}{0.001} & \multicolumn{2}{|c|}{$\frac{1}{<0001}$} & \multicolumn{2}{|c|}{$\frac{1}{40001}$} & \multicolumn{2}{|c|}{0.04} \\
\hline $\begin{array}{l}\text { Hypokinetic } \\
\text { arrhythmias } \\
\text { (\%) }\end{array}$ & 0.6 & 0.4 & 0.6 & 0 & 0.5 & 0.3 & 0 & 0.3 & 1.6 & 0.1 \\
\hline & \multicolumn{2}{|c|}{$\frac{1}{n 5}$} & \multicolumn{2}{|c|}{ ns } & \multicolumn{2}{|c|}{ ns } & \multicolumn{2}{|c|}{$\frac{1}{n s}$} & \multicolumn{2}{|c|}{ ns } \\
\hline $\begin{array}{l}\text { Pulmonary } \\
\text { hypertension } \\
\text { (\%) }\end{array}$ & 0.6 & 26 & 1.7 & 1.7 & 0.5 & 1.9 & 1.1 & 2.1 & 1.6 & 18 \\
\hline$P$ & \multicolumn{2}{|c|}{ ns } & & & \multicolumn{2}{|r|}{ ns } & \multicolumn{2}{|c|}{ ns } \\
\hline $\begin{array}{l}\text { Myocardial } \\
\text { fibrosis } \\
\text { (\$) }\end{array}$ & $\begin{array}{c}19 \\
(29 / 152)\end{array}$ & $\begin{array}{c}17 \\
(38 / 228)\end{array}$ & $\begin{array}{c}24 \\
(37 / 154)\end{array}$ & $\begin{array}{c}19 \\
(48 / 250)\end{array}$ & $\begin{array}{c}19 \\
(33 / 171)\end{array}$ & $\begin{array}{c}19 \\
(1211630)\end{array}$ & $\begin{array}{c}31 \\
(24 / 78)\end{array}$ & $\begin{array}{c}19 \\
\text { (114/610) }\end{array}$ & $\begin{array}{c}22 \\
(12 / 54)\end{array}$ & $\begin{array}{c}20 \\
(136,697)\end{array}$ \\
\hline$P$ & 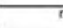 & & & & 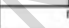 & 15 & & 012 & & ns \\
\hline
\end{tabular}

* near to the statistical significance

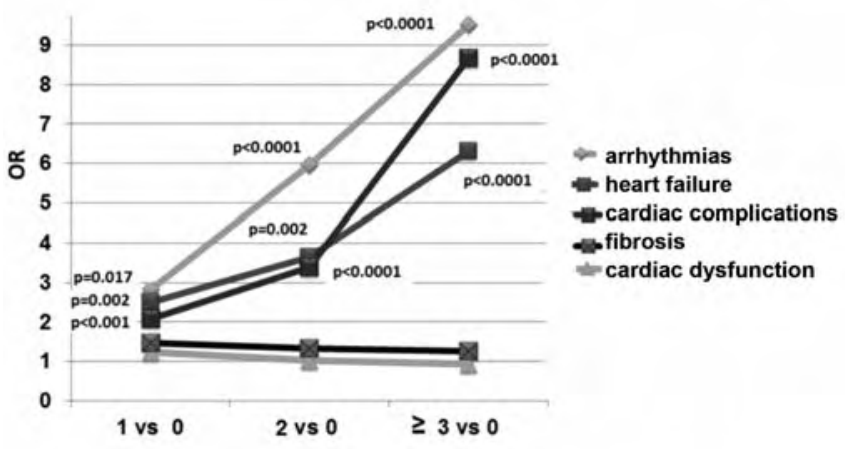

Figure 3. Risk of cardiac diseases in presence of one, two, three or more versus no endocrine diseases in patients with thalassemia major. and hypothyroidism didn't seem to play any role on the development of cardiac complications. Hypoparathyroidism appeared to be associated with a significant reduction of the risk of cardiac dysfunction. In this case, a role of vitamin D therapy can be supposed. Hypogonadism in males strongly increased the risk of arrhythmias. Males were expected to be more sensitive than females to iron damage ${ }^{17}$ and arrhythmias might be the consequence of a previous but resolved cardiac iron overload. However, the link between pubertal hypogonadism and arrhythmias has to be clarified. Diabetes increased the risk of arrhythmias as well as myocardial fibrosis, with a borderline effect on heart failure: a cardiomyopathy secondary to diabetes could be assumed.

This study presents some limitations. Secondary hypogonadism, subclinical hypothyroidism, hormone replacement therapy in hypogonadism, effects of sex on endocrinopathies out of hypogonadism were not taken into account. Besides this, growth hormone deficiency, reported prevalent up to the $40 \%$ of adult patients with TM, was not expected $^{18}$

Table 2. Prevalence of cardiac complications and myocardial fibrosis in patients with thalassemia major without myocardial iron overload ( $\mathrm{T}^{*}>20 \mathrm{~ms}$ in all segment) according to endocrinopathies.

\begin{tabular}{|c|c|c|c|c|c|c|c|c|c|c|}
\hline & \multicolumn{2}{|c|}{$\begin{array}{c}\text { Pubertal } \\
\text { hypogonadism } \\
\text { in males }\end{array}$} & \multicolumn{2}{|c|}{$\begin{array}{c}\text { Pubertal } \\
\text { hypogonadism } \\
\text { in females }\end{array}$} & \multicolumn{2}{|c|}{ Hypothyroidism } & \multicolumn{2}{|c|}{ Diabetes mellitus } & \multicolumn{2}{|c|}{ Hypoparathyroidism } \\
\hline & with & without & with & without & with & Without & with & without & with & without \\
\hline $\begin{array}{l}\text { Number of } \\
\text { patients }\end{array}$ & 70 & 137 & 62 & 130 & 75 & 333 & 29 & 329 & 24 & 353 \\
\hline $\begin{array}{l}\text { Cardiac } \\
\text { dysfunction } \\
\text { (\%) }\end{array}$ & 43 & 37 & 18 & 20 & 33 & 28 & 45 & 28 & 13 & 32 \\
\hline$p$ & \multicolumn{2}{|c|}{ ns } & \multicolumn{2}{|c|}{ ns } & \multicolumn{2}{|c|}{ ns } & \multicolumn{2}{|c|}{0.061} & \multicolumn{2}{|c|}{0.065} \\
\hline $\begin{array}{l}\text { Heart failure } \\
\text { (by MRs and by } \\
\text { patiensthisiory } \\
\text { present } n \text { the } \\
\text { time of the } \\
\text { study) } \\
\text { (\%) }\end{array}$ & 17 & 11 & 7 & 4 & 13 & 8 & 24 & 8 & 13 & 9 \\
\hline P & \multicolumn{2}{|c|}{ ns } & \multicolumn{2}{|c|}{ ns } & \multicolumn{2}{|c|}{ n.5 } & \multicolumn{2}{|c|}{6.004} & \multicolumn{2}{|r|}{$n$} \\
\hline $\begin{array}{l}\text { Hyperkinetic } \\
\text { arrhythmias } \\
(\%)\end{array}$ & 24 & 1.5 & 10 & 2 & 16 & 5 & 35 & 5 & 21 & 6 \\
\hline$p$ & \multicolumn{2}{|c|}{$<0.0001$} & \multicolumn{2}{|c|}{0.015} & \multicolumn{2}{|c|}{$<0.0001$} & \multicolumn{2}{|c|}{$<0.0001$} & \multicolumn{2}{|c|}{0.021} \\
\hline $\begin{array}{l}\text { Myocardial } \\
\text { fibrosis } \\
\text { (\%) }\end{array}$ & $\begin{array}{c}19 \\
\text { (1265) }\end{array}$ & $\begin{array}{c}17 \\
\text { (19/111) }\end{array}$ & $\begin{array}{c}30 \\
\text { (17/56) }\end{array}$ & $\begin{array}{c}19 \\
\text { (21/113) }\end{array}$ & $\begin{array}{c}23 \\
(14 / 61)\end{array}$ & $\begin{array}{c}19 \\
(56 / 293)\end{array}$ & $\begin{array}{c}38 \\
(9 / 24)\end{array}$ & $\begin{array}{c}20 \\
(56 / 287)\end{array}$ & $\begin{array}{c}29 \\
(6 / 21)\end{array}$ & $\begin{array}{l}20 \\
(136 / 697)\end{array}$ \\
\hline$p$ & & 75 & & ns & & ns & & .037 & & ns \\
\hline
\end{tabular}

* near to the statistical significance

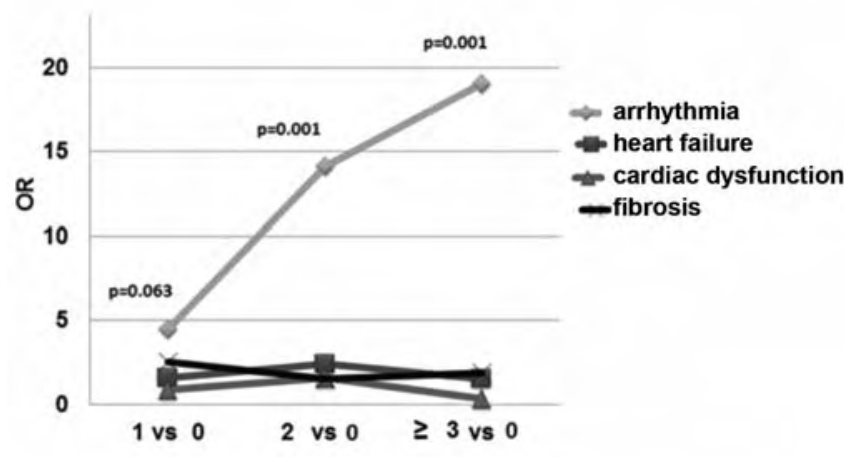

Figure 4. Risk of cardiac diseases in presence of one, two, three or more versus no endocrine diseases in patients with thalassemia major without myocardial iron overload. 
Table 3. OR for cardiac complications and myocardial fibrosis in patients with thalassemia major without myocardial iron overload according to endocrinopathies.

\begin{tabular}{|c|c|c|c|c|c|c|c|c|}
\hline & & & \multicolumn{6}{|c|}{ Adjusted for } \\
\hline & & & \multicolumn{2}{|c|}{ age } & \multicolumn{2}{|c|}{ Endocrinopathy } & \multicolumn{2}{|c|}{ Both } \\
\hline & OR & $\mathbf{p}$ & OR & $\mathbf{p}$ & OR & $\mathbf{p}$ & OR & $\mathbf{p}$ \\
\hline \multicolumn{9}{|c|}{ Hypogonadism in males } \\
\hline Arrhythmias & 21.65 & $<0.0001$ & 17.25 & $<0.0001$ & 17,16 & $<0.0001$ & 17.21 & 0.0001 \\
\hline \multicolumn{9}{|c|}{ Hypogonadism in females } \\
\hline Arrhythmias & 6.86 & 0.021 & 3.72 & ns & 8.09 & 0.070 & 2.24 & ns \\
\hline \multicolumn{9}{|c|}{ Hypothyroidism } \\
\hline Arrhythmias & 4.04 & 0.001 & 2.86 & 0.018 & 2.14 & ns & 1.36 & ns \\
\hline \multicolumn{9}{|c|}{ Diabetes mellitus } \\
\hline Arrhythmias & 9.66 & $<0.0001$ & 3.75 & $<0.0001$ & 7.28 & $<0.0001$ & 3.5 & 0.015 \\
\hline Heart failure & 3.78 & 0.006 & 2.02 & ns & 3.21 & 0.017 & 1.94 & ns \\
\hline Fibrosis & 2.46 & 0.043 & 2.31 & ns & 2.14 & ns & 2.12 & 0.017 \\
\hline \multicolumn{9}{|c|}{ Hypoparathyroidism } \\
\hline Arrhythmias & 3.96 & 0.012 & 2.52 & ns & 1.99 & ns & 1.59 & ns \\
\hline $\begin{array}{l}\text { LV/LR } \\
\text { dysfunction }\end{array}$ & 0.31 & $\cdot 0.060$ & 0.24 & 0.025 & 0.28 & 0.043 & 0.24 & 0.027 \\
\hline
\end{tabular}

* near to the statistical significance

\section{References}

1. Aessopos A, Berdoukas V. Cardiac function and iron chelation in thalassemia major and intermedia: a review of the underlying pathophysiology and approach to chelation management. Mediterr J Hematol Infect Dis 2009;1:e2009002.

2. Wood JC. Cardiac complications in thalassemia major. Hemoglobin 2009;33:S81-86.

3. Gamberini MR, De Sanctis V, Gilli G. Hypogonadism, diabetes mellitus, hypothyroidism, hypoparathyroidism: incidence and prevalence related to iron overload and chelation therapy in patients with thalassaemia major followed from 1980 to 2007 in the Ferrara Centre. Pediatr Endocrinol Rev 2008;6:158-169.

4. Wood JC, Enriquez C, Ghugre N, et al. Physiology and pathophysiology of iron cardiomyopathy in thalassemia. Ann N Y Acad Sci 2005;1054:386-395.

5. De Sanctis V, Govoni MR, Sprocati M, Marsella M, Conti E. Cardiomyopathy and pericardial effusion in a 7 year-old boy with $\beta$ thalassaemia major, severe primary hypothyroidism and hypoparathyroidism due to iron overload. Pediatr Endocrinol Rev 2008;6:181-184.

6. Erfurth EM, Holmer H, Nilsson PG, Kornhall B. Is growth hormone deficiency contributing to heart failure in patients with $\beta$-thalassemia major? Eur J Endocrinol 2004;151:161-166.
7. Meloni A, Ramazzotti A, Positano V, et al. Evaluation of a web-based network for reproducible T2* MRI assessment of iron overload in thalassemia. Int J Med Inform 2009;78:503-512.

8. Ramazzotti A, Pepe A, Positano V, et al. Multicenter validation of the magnetic resonance $t{ }^{*}$ technique for segmental and global quantification of myocardial iron. J Magn Reson Imaging 2009;30:62-68.

9. Pepe A, Positano V, Santarelli F, et al. Multislice multiecho T2* cardiovascular magnetic resonance for detection of the heterogeneous distribution of myocardial iron overload. J Magn Reson Imaging 2006;23:662-668.

10. Pepe A, Lombardi M, Positano V, et al. Evaluation of the efficacy of oral deferiprone in $\beta$-thalassemia major by multislice multiecho T2*. Eur J Haematol 2006;76:183-192.

11. Cerqueira MD, Weissman NJ, Dilsizian V, et al. Standardized myocardial segmentation and nomenclature for tomographic imaging of the heart: a statement for healthcare professionals from the Cardiac Imaging Committee of the Council on Clinical Cardiology of the American Heart Association. Circulation 2002;105:539-542.

12. Pepe A, Positano V, Capra M, et al. Prevalence and clinicalInstrumental correlates of myocardial scarring by delayed enhancement cardiovascular magnetic resonance in thalassemia major. Heart 2009;95:1688-1693.

13. Anderson LJ, Holden S, Davis B, et al. Cardiovascular T2-star (T2*) magnetic resonance for the early diagnosis of myocardial iron overload. Eur Heart J 2001;22:2171-2179.

14. Meloni A, Del'Amico M, Favilli B, et al. Right Ventricular Volumes and Function normalized to body surface area, age and sex in a large cohort of well-treated Thalassemia Major without myocardial iron overload. Journal of Cardiovascular Magnetic Resonance; 13:P299.

15. Meloni A, Dell'Amico M, Favilli B, et al. Left Ventricular Volumes, Mass and Function normalized to the body surface area, age and gender from CMR in a large cohort of well-treated Thalassemia Major patients without myocardial iron overload. Journal of Cardiovascular Magnetic Resonance; 13:P305.

16. Cogliandro T, Derchi G, Mancuso L, et al. Guideline recommendations for heart complications in thalassemia major. J Cardiovasc Med (Hagerstown) 2008;9:515-525.

17. Marsella M, Borgna-Pignatti C, Meloni A, et al. Cardiac iron and cardiac disease in males and females with transfusion-dependent thalassemia major: a $\mathrm{T} 2 *$ magnetic resonance imaging study. Haematologica;96:515-520.

18. Scacchi M, Danesi L, Cattaneo A, et al. Growth hormone deficiency (GHD) in adult thalassaemic patients. Clin Endocrinol (Oxf) 2007;67:790-795. 